\title{
The Management and Care of the Type 1 Diabetic Athlete
}

\author{
Willingham $\mathrm{E}^{*}$
}

\author{
Master of Science in Nutrition, Healthspan, and Longevity Candidate, University of Southern California, United \\ States
}

*Corresponding author: Willingham E, Master of Science in Nutrition, Healthspan, and Longevity Candidate, University of Southern California, United States, United States, E-mail: ewilling@usc.edu

Citation: Willingham E (2017) The Management and Care of the Type 1 Diabetic Athlete. J Nutr Health Sci 4(2): 204. doi: 10.15744/2393-9060.4.204

Received Date: May 17, 2017 Accepted Date: July 05, 2017 Published Date: July 07, 2017

\begin{abstract}
Diabetes poses many unique challenges to all individuals affected by the disease, particularly the diabetic athlete due to the effect that exercise has on blood glucose levels within the body. Patients and professionals must demonstrate significant understanding of the complex interaction of many physiological and environmental components that contribute to the maintenance of glucose homeostasis, or lack thereof. Type 1 Diabetes Mellitus is the form of Diabetes most often seen in elite athletes. In order for these athletes to not only normalize, but also maximize athletic performance, the healthcare and athletic professionals managing the athlete must understand and apply how blood glucose levels, hormones, environment, and physical activity interact to prevent hypoglycemia and hyperglycemia, conditions that threaten the athlete's performance as well as their overall general health.
\end{abstract}

Keywords: Type 1 Diabetes; Athlete; Medical Review

\section{Introduction}

Diabetes Mellitus is a chronic endocrine disorder characterized by hyperglycemia. Type 1 Diabetes Mellitus (T1DM) is the rarer form of the disease, affecting about 5-7\% of the diabetic population, but it is more likely to be seen in athletes than Type 2 Diabetes. T1DM is an autoimmune disorder that results from a combination of genetic and unknown environmental factors. An absolute insulin deficiency characterizes T1DM [1]. Damage to beta cells in the pancreas causes an absence in insulin production. Since the pancreas of a diabetic fails to produce insulin, blood glucose levels go unregulated, back and forth between either hypoglycemia or hyperglycemia. This typically leads to "common pathophysiologic complications associated with T1DM, such as neuropathy, nephropathy, retinopathy, and cardiopulmonary dysfunction" [2]. Since the normal mechanism of glucose homeostasis maintenance is lost, it "must be appropriated with exogenous insulin in order to approximate normal physiology", as well as normalize and enable maximum athletic performance [3,4]. Prior to the advent of exogenous insulin, "patients with T1DM had little choice but to limit themselves from participation in physical activity, as metabolic disturbances complicate significant exercise" [5].

Now, health professionals accept and generally recommend exercise for the management of T1DM and for an improvement in overall quality of life in affected individuals, so physicians should encourage the physical activity required of athletes [6]. Many doctors find it remarkable that people with T1DM are able to compete at the highest level, yet doctors encourage competition and training, as "regular routine exercise generally improves glucose uptake by increasing insulin sensitivity and decreasing body adiposity levels [3]". Athletes with T1DM who experience the metabolic disturbances unique to the disease can benefit from a well-organized plan that may allow them to compete on an even field with their competitors who do not have to rely on exogenous insulin for their bodies to function normally and perform at their maximum abilities [1].

Patients and professionals must understand how exercise intensity and duration influence fuel sources- at rest, the body uses free fatty acids, but during the transition to exercise the emphasis switches to carbohydrate (in the form of glycogen and glucose). Because the body of a Type 1 diabetic does not possess the ability to reduce circulating insulin at the onset of exercise, the result is a hypoglycemic state due to the imbalance in glucose uptake by skeletal muscles. "When insulin is not available to assist in the transport of glucose into skeletal muscles during exercise, glucose uptake decreases, glucose release from the liver increases", and there is a subsequent increase in blood glucose [7]. "Without adequate insulin to cover this imbalance, skeletal muscles are forced 
to rely on fat as fuel", which potentially leads to the development of ketone bodies that damage the liver [7].

Managing diabetes within the context of sport and exercise is a challenging but increasingly important skill- professionals "must understand the body's hormonal milieu as well as take into account the type and duration of an athlete's physical activity". The demands of physical activity and competition make it more challenging to care for the diabetic athlete than just the regular diabetic patient, but it is crucial to keep blood glucose levels at or as close to normal as possible pre-exercise, so that an athlete does not become hypoglycemic or hyperglycemic $[1,4]$. While both are detrimental to an athlete's health and performance, "hypoglycemia is more likely to prevent an athlete from competing than hyperglycemia" [8].

\section{General Management}

In managing the athlete with T1DM, the most important goal is to keep blood glucose levels at or as close to normal levels as possible without causing hypoglycemia, which requires the maintenance of a delicate balance among hypoglycemia, euglycemia, and hyperglycemia. Due to the demands of physical activity and competition, this balance is significantly more difficult to achieve in an athlete [1]. Athletic professionals should work with the T1DM athlete to come up with an extensive personalized diabetes care plan that recognizes the necessary supplies for athletic training kits, participation in physical exams, treatment and prevention of hyperglycemia or hypoglycemia, insulin administration, travel recommendations, athletic injury protocol, and management of glycemic control. Blood glucose monitoring guidelines should include the frequency of monitoring, insulin therapy guidelines, emergency contact information, a medic alert plan, supplies for urine and blood ketone testing, a "sharps" container, spare batteries, and checked expiration dates of all supplies. Professionals should have a "specific care plan for practice and games that identifies blood glucose targets as well as symptoms of and strategies to prevent exercise associated hypoglycemia, hyperglycemia, and ketosis." A study of football players that have T1DM revealed that "setting target blood glucose levels between 150 and $250 \mathrm{mg} /$ $\mathrm{dl}$ helped authors to limit hypoglycemia during football games." Such discoveries are helpful for professionals to keep in mind as a ballpark number or to use as a potential guideline, especially when working with football players [7]. Ideally, blood glucose should be monitored and recorded before and after each meal, as well as before, during, and after each training session or athletic contest, although this depends on the type, duration, and intensity of exercise. It is crucial that an elite T1DM athlete "establishes a habitual training program that mimics competition." If exercise or training is unplanned or unexpected, "insulin dosage adjustment will be impossible and a carbohydrate supplement will be necessary", also depending on the timing and duration of the exercise [3].

During competition, blood sugar should be checked at changeovers or halftime. It is possible that insulin injection is necessary. If so, insulin should be administered into subcutaneous tissue- intramuscular injection sites should be avoided, as muscle contractions may accelerate insulin absorption [1]. Effective insulin injection does not only rely on the injection site, but can also rely on factors external to the athlete's body. Athletes and professionals should be wary of travel to different time zones, as insulin therapy may need to be adjusted to coordinate changes in eating and activity patterns. Long term success and management of insulin injection and glycemic control is determined by a "glycosylated hemoglobin (HbA1c) assay that should occur every 3-4 months, but this number is not valuable for every day decisions". The American Diabetes Association recommends a HbA1c level of 7\%, and in general a lower HbA1c correlates with a lower risk of complications, at the expense of increased hypoglycemic risk.

\section{Hypoglycemia}

\section{Internal Risk Factors}

Glycemia during exercise varies within an individual diabetic, as well as amongst the diabetic population, depending on factors such as exercise modality intensity, nutritional status, time of insulin injection, or pre-exercise glycemia level. "The blood glucose lowering effect of moderate intensity exercise increases the risk of developing an episode of hypoglycemia." Hypoglycemia is the most severe acute complication of intensive insulin therapy, and exercise is the most direct common cause [1,6]. Hypoglycemia is "a result of overinsulinization both during and after exercise"- the rate at which the body absorbs subcutaneously injected insulin increases with exercise due to an increase in body temperature and in subcutaneous muscle blood flow. Hypoglycemia can also result from an impaired release of counter regulatory hormones caused by a previous bout of exercise or a previous hypoglycemic episode. Type 1 Diabetics have a greater predisposition to hypoglycemia 20-30 minutes after beginning exercise, as “insulin levels do not reduce upon beginning exercise, but as exercise promotes blood flow to and absorption from depots in subcutaneous tissues, then insulin levels begin to increase" [8].

There is increased potential for hypoglycemia to develop during endurance exercise that lasts several hours. There is additional increased risk for males and adolescents. Additionally, hypoglycemic symptoms may be worse in the morning [3]. Physical activity combined with the stress of competition often causes symptoms of hypoglycemia to manifest, which then promotes glucose production and antagonizes insulin production [9]. It is crucial for healthcare professionals to understand how physiological and environmental factors combine and become significantly magnified in the T1DM individual when exercising to produce hypoglycemia, and develop specific protocol to prevent it.

\section{Treatment/Management Plan}

In order to prevent hypoglycemia in moderately intense activities, athletic professionals working with the T1DM athlete should 
advise the athlete to reduce the insulin dosage by $50-90 \%$ prior to activity [8]. Eating a low glycemic index pre-exercise meal is a good recommendation for athletes and may help improve performance, while high glycemic index foods give more immediate acting carbohydrate and can be used instead of or in conjunction with a sports drink before, during, or immediately following exercise to prevent hypoglycemia. Sports drinks are excellent sources of fast acting carbohydrates and electrolytes that are necessary when an athlete's blood sugar drops undesirably. Whenever hypoglycemia does occur during sports, current guidelines recommend stopping exercise, treating the athlete with 15 grams of a fast acting carbohydrate to increase blood glucose, waiting 15 more minutes, then retesting again and treating the athlete further if euglycemia is not restored [9].

A study that explores the effect of different intensities of exercise on the diabetic athlete revealed that a brief 'sprint' or series of intermittent high intensity exercise bouts, is associated with less of a risk for late-onset post exercise hypoglycemia, and can be used to prevent hypoglycemia [10]. Additional unconventional treatment for hypoglycemia includes the ingestion of $5 \mathrm{mg} / \mathrm{kg}$ of caffeine prior to exercise, which has been shown to reduce hypoglycemia during and following exercise [4].

\section{Hyperglycemia}

\section{Internal Risk Factors}

Exercise is a metabolic challenge and in response, the endocrine system produces a synchronized reaction. As muscle glycogen stores deplete, "a balance develops between glucose production and glucose uptake by the exercising muscle." In non-diabetic patients, "during exercise, circulating insulin concentrations fall, but an increased secretion of counter regulatory hormones such as adrenaline, glucagon, cortisol, and growth hormone increase glucose production" [4]. Once exercise stops, circulating catecholamine levels rapidly fall and insulin levels rapidly rise [3]. As a result, hyperglycemia and hyperinsulinemia combine postexercise to provide homeostatic metabolic conditions for replenishment of muscle glycogen necessary for recovery [4]. The body then "enters a fasted state in which glycogen stores in the muscle and liver are low and hepatic glucose production is high" [3].

The signs and symptoms of initial T1DM diagnosis develop rapidly and relate to hyperglycemia, including frequent urination, thirst, hunger and polyphagia, weight loss, visual disturbances, fatigue, and ketosis. As mentioned above, the psychological stress associated with competition frequently associated with increases in blood glucose levels before competition also causes an increase in counter regulatory hormone levels. This increase in blood glucose concentrations is manifested symptomatically in the form of nausea, dehydration, decreased cognitive performance, slowing of visual reaction time, feeling sluggish, and fatigue [1]. Injury is also associated with increases in the secretion of these stress hormones and can also cause an increase in blood glucose levelsan exaggerated hyperglycemic response in people with T1D. Therefore, an increased risk of infection as well as poor wound and fracture healing associates with poor blood glucose regulation. All of these associations are vital for athletes with T1DM and health professionals to look out for when a patient is involved in training or competition, and "an individualized blood glucose management protocol should be developed for use during injury and recovery". If untreated, chronic hyperglycemia leads to long term damage, dysfunction, and failure of various organs- particularly the eyes, kidneys, nerves, and heart [1]. Additional symptoms manifest as tachycardia, sweating, palpitations, hunger, nervousness, headache, trembling, and dizziness. The brain neuronal glucose deprivation of hyperglycemia can also cause blurred vision, fatigue, difficulty thinking, aggressive behavior, seizures, convulsions, or loss of consciousness- all symptoms that could severely hinder an athlete's well-being in training, competition, and performance.

The chronic high blood glucose of hyperglycemia causes chemical changes in the nerves and damages blood vessels that carry oxygen and nutrients to the nerves [11]. Hyperglycemia increases the risk for microvascular, macrovascular, and neuropathic complications. "Bilateral sensory involvement with a dull perception of vibration, pain, and temperature, particularly in low extremities, characterizes peripheral neuropathy," which is a particular concern for the T1DM athlete and this is even more of a concern for athletes performing weight bearing activities [1].

\section{Treatment/Management Plan}

Arguably one of the most important components in caring for an athlete with T1DM is not only managing blood glucose levels, but also tracking other long-term side effects of T1DM. These are most often a resultof chronic hyperglycemia, such as diabetic neuropathy. The team management approach to caring for an athlete with T1DM is well established and supported in health literature, because the teammay require specialists that can provide the best care for an athlete and long term T1DM related complications [1]. The long term T1DM related complications more frequently result from hyperglycemia than hypoglycemia. For example, Type 1 Diabetics should be screened annually for cardiovascular disease, retinopathy, nephropathy, neuropathy, and a foot exam to check sensory function and ankle reflexes. Additionally, a "graded stress test should be given to evaluate potential cardiovascular dysfunction". The ADA recommends an initial dilated and comprehensive ophthalmologic exam 3-5 years after diagnosis, and an annual screening thereafter for retinopathy, glaucoma, and cataracts. "Feet should be inspected regularly for neuropathic symptoms such as reddened areas, blisters, abrasions, or lacerations". Specialist like ophthalmologists and podiatrists are required components of the "team" in order to get these screenings. By working together, the diabetic management team help to prevent and treat immediate and outstanding complications that result from chronically high blood glucose levels, which enhances an athlete's health and performance. 


\section{External Risk Factors}

One health risk that is especially unique to athletes with T1DM is the fact that they can essentially control their own blood glucose levels, and may resort to a number of practices that compromise blood glucose control and general health in an attempt to gain a competitive advantage. This includes unsafe dietary patterns or the omission of insulin injections, which is especially common in sports that have weight categories [7]. A study of weight control practices in athletes with T1DM reports unhealthy weight control by using a method of skipping insulin, or taking less insulin in an effort to control body weight. Skipping insulin injections results in hyperglycemia and the associated symptoms described above, such as rapid weight loss. When athletes with T1DM engage in this type of behavior, they automatically risk their health and athletic performance. Trainers and coaches, specifically those working with athletes that participate in sports with weight categories, or sports where appearance is emphasized, should be aware of the potential for their T1DM athlete to engage in such practices and what those risky behaviors look like.

An additional external challenge for the T1DM athlete, is environment and climate. The cold temperature "increases glucose uptake to generate heat in muscles, and altitude by increased reliance on carbohydrate fuel sources due to decreased oxygen tension" - both extremities that result in more glucose circulating in the blood and a subsequent hyperglycemic response [8]. Autonomic neuropathy may affect gastrointestinal, neuroendocrine, and cardiovascular systems. In terms of the cardiovascular system, neuropathy causes a decrease in nitric oxide, which may lead to constriction of blood vessels that supply the nerves in diabetic patients. "Constriction subsequently impairs the nerves ability to transmit signals", and this condition can be magnified in the cold [11]. Patients with "any form of diabetic neuropathy may have an increased risk of injury from falling secondary to instability and reduced muscle strength." Once again, any athletic professional working with a T1DM athlete should be cognizant of these signs and symptoms, as they will impair the athlete's performance and are dangerous to their health [3].

\section{Conclusion}

When tailoring advice to individuals with T1DM, athletic professionals must consider factors that mostly revolve around the type, duration, and intensity of exercise. These factors will influence a T1DM athlete's reaction to the exercise, and the type of treatment that he or she needs. Professionals must be aware of such specific details regarding the interaction of these three factors and subsequent treatment. Examples include the type and amount of carbohydrate supplement a diabetic might need, the fact that lower intensity exercise is more likely to contribute to hyperglycemia than higher intensity exercise, the risk of delayed post exercise hypoglycemia, and precautionary measures for hyperglycemia and hypoglycemia [4]. If such factors are not taken into consideration and specified when fueling and caring for the athlete, he or she might have a less successful athletic performance and experience detrimental internal health effects.

\section{References}

1. Jimenez CC, Corcoran MH, Crawley JT, Hornsby WG, peer KS et al. (2007) National Athletic Trainers' Association Position Statement: Management of the Athlete with Type 1 Diabetes Mellitus. J Athl Train 42: 536-45.

2. Wheatley CM, Baldi JC, Cassuto NA, Foxx-Lupo WT, Snyder EM (2011) Glycemic control influences lung membrane diffusion and oxygen saturation in exercise-trained subjects with type 1 diabetes: alveolar-capillary membrane conductance in type 1 diabetes. Eur J Appl Physiol 111: 567-78.

3. Peirce NS (1999) Diabetes and exercise. Br J Sports Med 33:161-73.

4. Gallen IW, C Hume, A Lumb (2011) Fuelling the athlete with type 1 diabetes. Diabetes Obes Metab 13: 130-6.

5. Steppel JH, Horton ES (2003) Exercise in the Management of Type 1 Diabetes Mellitus. Rev Endocr Metab Disord 4: 355-60.

6. Tonoli C, Heyman E, Roelands B, Buyse L, Cheung SS et al. (2012) Effects of Different Types of Acute and Chronic (Training) Exercise on Glycaemic Control in Type 1 Diabetes Mellitus: a meta-analysis. Sports Med 42: 1059-80.

7. Hornsby W Guyton, Robert D Chetlin (2005) Management of Competitive Athletes WIth Diabetes. Diabetes Spectrum 18: $102-07$.

8. Perry E, IW Gallen (2009) Guidelines on the current best practice for the management of type 1 diabetes, sport and exercise. Pract Diab 26: 116-23.

9. Riddell MC, Milliken J (2011) Preventing Exercise-Induced Hypoglycemia in Type 1 Diabetes Using Real-Time Continuous Glucose Monitoring and a New Carbohydrate Intake Algorithm: An Observational Field Study. Diabetes Technol Ther 13: 819-25.

10. Iscoe KE, MC Riddell (2011) Continuous moderate-intensity exercise with or without intermittent high-intensity work: effects on acute and late glycaemia in athletes with Type 1 diabetes mellitus. Diabet Med 28: 824-32.

11. Kaur Prabhjot, Maman Paul, Jaspal Singh Sandhu (2006) Auditory and visual reaction time in athletes, healthy controls, and patients of type 1 diabetes mellitus: A comparative study. Int J Diab Dev Ctries 26: 112-15. 


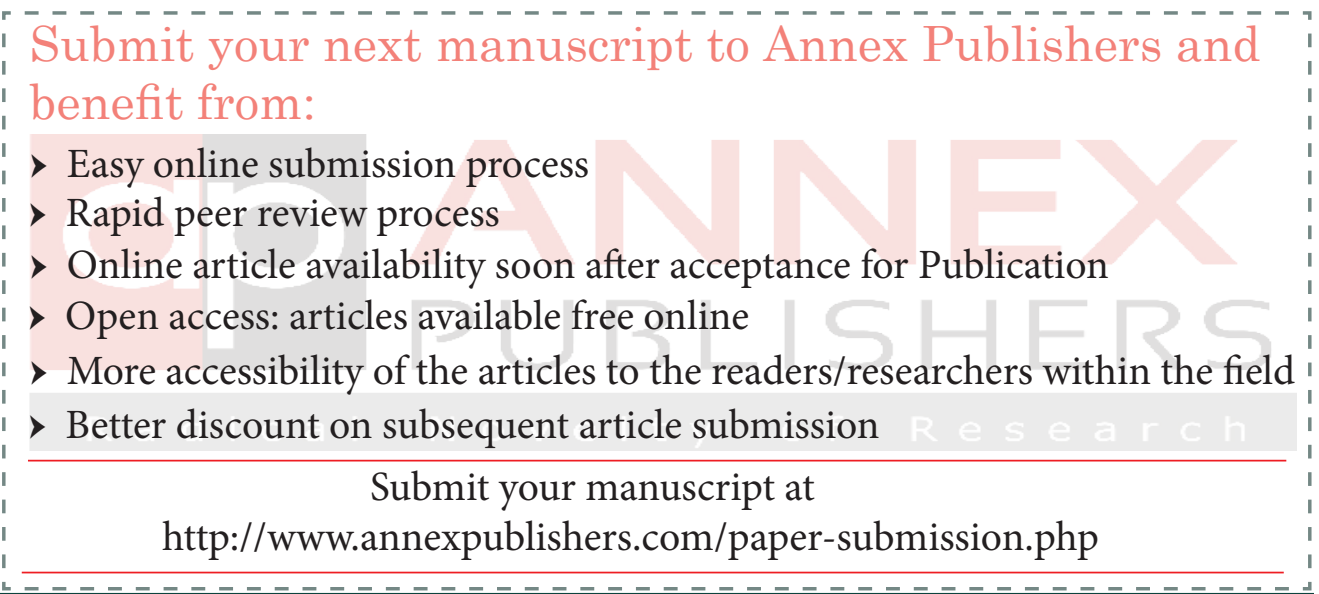

